\title{
Evaluation agromorphologique et sélection des meilleures accessions de Pourghère (Jatropha curcas L.) introduites au Bénin
}

\author{
L. E. AHOTON ${ }^{1 *}$, F. QUENUM $^{1}$, et G. MERGEAI ${ }^{2}$ \\ ${ }^{1}$ Faculté des Sciences Agronomiques, Université d'Abomey-Calavi, Bénin. \\ ${ }^{2}$ Département des sciences et production du vivant, Unité de Phytotechnie tropicale et horticulture, Université \\ de Liège Belgique. \\ *Corresponding author, E-mail: essehahoton@yahoo.fr, BP 03255 Cotonou 03, Bénin.
}

\section{RESUME}

Jatropha curcas est un arbuste à usages multiples très connu des populations africaines. Il est cultivé comme une plante médicinale dans plusieurs pays tropicaux et subtropicaux. Ses graines contiennent de l'huile qui peut être transformée en biocarburant. Les caractéristiques agromorphologiques de huit accessions nouvellement introduites au Bénin et un écotype local ont été étudiées à partir de douze caractères qualitatifs et quantitatifs. Une analyse de la variance a été réalisée en vue de ressortir les différences entre accessions. Les résultats obtenus montrent que la provenance Equateur (Salinas) a la hauteur la plus élevée, le diamètre au collet le plus grand et les feuilles les plus développées. Par contre, la provenance Sénégal a le nombre de ramifications le plus élevé, les feuilles les plus réduites et la production en fruits la plus importante, compte tenu du fait que les ramifications fructifient au fur et à mesure de leur apparition. Il existe une différence très hautement significative (seuil $0,1 \%$ ) en ce qui concerne le diamètre au collet, la largeur et la longueur des feuilles. Les caractères agromorphologiques évalués ont permis d'identifier parmi les provenances cultivées, les écotypes pouvant rentrer dans un programme d'amélioration et de sélection variétale en vue de mettre à la disposition des producteurs de nouveaux cultivars plus performants et plus précoces.

(C) 2011 International Formulae Group. All rights reserved.

Mots clés : Jatropha curcas, écotypes, caractères agromorphologiques, Bénin.

\section{INTRODUCTION}

Jatropha curcas, arbuste à usages multiples, connu sous les appellations de pignon d'Inde ou pourghère, appartient à la famille des Euphorbiacées et au genre Jatropha. Il est originaire du Mexique et de l'Amérique Centrale (Grim, 1999). Actuellement, cet arbuste est largement cultivé dans plusieurs autres pays de l'Amérique Latine, d'Asie, et d'Afrique. Le genre compte présentement environ 170 espèces connues et répertoriées (Makkar et Becker, 1999). En pays tropicaux, cet arbuste est connu pour ses usages médicinaux et pour son huile. Il est utilisé pour traiter la dermatose, la toux, le rhumatisme, l'arthrite, les coliques, et la jaunisse (Staubmann et al., 1997). Au Bénin, les différentes parties de cet arbuste sont employées dans la lutte contre l'hypertension, les plaies, le diabète, les fractures, les courbatures, etc. (de Souza, 2005). Selon Arbonnier (2002), les feuilles et 
les jeunes tiges sont également utilisées contre la fièvre, la syphilis, la malaria, l'ictère, les infections buccales, les douleurs, les rhumatismes etc. Au Mali, l'huile extraite des graines est utilisée comme biocarburant pour les moulins à grains, les motopompes, les groupes électrogènes, et comme lubrifiant pour les moteurs (Henning, 1997 ; Gübitz et al., 1999). Les extraits issus de la graine et de la feuille de $J$. curcas ont des propriétés mollucicides, fongicides et insecticides (Nwosu et Okafor, 1995 ; Liu et al., 1997 ; Solsoloy et Solsoloy, 1997).

Sur le plan agricole, J. curcas est résistant à la sécheresse et peut valoriser 250 $\mathrm{mm}$ d'eau annuellement pour son développement (Makkar et Becker, 1999). C'est un arbuste qui peut être cultivé sur des terres incultes, pauvres, marginales où il n'existe aucune possibilité d'irrigation. C'est un arbuste peu exigeant. Il peut être utilisé pour lutter contre l'érosion et dans la confection des haies vives. Son utilisation comme engrais vert pour l'amélioration des sols acides a été rapportée par Scherchan et al. (1989).

La graine contient $55-60 \%$ d'huile qui peut être transformée en biocarburant et 30 à $32 \%$ de protéines (Ajiwe et al., 1996; Aderibigbe et al., 1997 ; Makkar et al., 1997). Malgré les multiples caractères que possède $J$. curcas, pour être en mesure de répondre à l'évolution des besoins du marché, il est nécessaire de créer de nouvelles variabilités. Pour ce faire, l'évaluation et la caractérisation des ressources génétiques sont les éléments déterminants dans les programmes d'amélioration et de sélection. L'objectif du présent travail est l'évaluation agromorphologique des accessions de $J$. curcas nouvellement introduites au Bénin en vue d'utiliser les meilleures accessions dans un programme d'amélioration génétique.

\section{MATERIEL ET METHODES \\ Zone d'étude}

L'essai a été conduit à la Ferme d'Application et de Production (FAP) de la Faculté des Sciences Agronomiques (FSA, Altitude $\left.17,4 \mathrm{~m}, 06^{\circ} 24^{\prime} \mathrm{N}, 02^{\circ} 20^{\prime} \mathrm{E}\right) \mathrm{de}$ l'Université d'Abomey-Calavi (UAC) de juin 2008 à mai 2009, située dans la commune
d'Abomey-Calavi (Bénin). Le climat dans la zone est de type subéquatorial et est caractérisé par deux saisons de pluies (avril à mi-juillet et septembre à novembre), et deux saisons sèches (décembre à mars et mi-juillet à septembre) avec des fluctuations ces dernières années. Le sol, communément appelé terre de barre est de nature ferralitique avec de la kaolinite en proportion non négligeable.

\section{Matériel végétal}

Les accessions de J. curcas nouvellement introduites au Bénin ont été fournies par le Prof. G. Mergeai du Département des Sciences et Production du Vivant, Unité de Phytotechnie Tropicale et Horticulture, Université de Liège Belgique. Ces accessions proviennent de l'Asie, de l'Amérique et de l'Afrique (Tableau 1). Une variété locale complète les accessions fournies par Liège. Les différentes périodes de récolte de chaque accession sont présentées dans le Tableau 1.

\section{Evaluation agromorphologique}

L'essai a été implanté sur une superficie de $400 \mathrm{~m}^{2}$. La distance entre plants et entre lignes est de $2 \mathrm{~m}$. Les graines scarifiées sont d'abord semées dans des sachets en polyéthylène de 18 x 10 x $8 \mathrm{~cm}^{3}$ et les plantules issues des graines germées ont été transplantées sur ladite parcelle en juin 2008. L'entretien du site expérimental a été fait régulièrement. Durant toute la période de la saison sèche, les plants ont été arrosés copieusement tous les jours et de préférence les après-midi. Les données collectées sur cinq (5) plants de chaque accession un an après plantation de $J$. curcas concernent les caractères quantitatifs et qualitatifs. Caractères quantitatifs : hauteur de la plante, diamètre au collet de la tige, longueur et largeur de dix (10) feuilles complètement ouvertes et aléatoirement choisies au sommet de la tige principale, le poids moyen de 10 graines choisies aléatoirement, le nombre de ramifications et le nombre de lobes. Caractères qualitatifs : forme des feuilles, couleur de la feuille, couleur de la tige, couleur des graines et couleur du pétiole. 


\section{Analyse des données}

Les valeurs moyennes des différents paramètres étudiés ont été calculées. La différence des valeurs notées est évaluée par une analyse de la variance à un facteur (ANOVA) et le test de Student, Newman et Keuls au seuil de $5 \%$ a été utilisé pour le classement des moyennes. L'analyse de la variance a été réalisée avec le logiciel SAS (version 9.1).

\section{RESULTATS}

\section{Germination des différentes accessions}

Le taux de germination varie d'une accession à une autre (Tableau 2). Ce taux est élevé lorsque la durée de conservation n'est pas trop longue. Toutes les graines récoltées en 2008 ont des pourcentages de germination assez élevés (au moins 87\%). Par contre, les graines récoltées entre 2006 et 2007 ont des taux de germination faibles à part les accessions de Cambodge et Kivu qui ont un pourcentage respectif de germination de $87 \%$ et $67 \%$. Ceci montre que le taux de germination est fonction de la durée et des méthodes de conservation. D'une manière générale, le taux de germination diminue en fonction de la durée de conservation.

\section{Hauteur des plantes}

L'analyse statistique montre qu'il existe une différence significative entre les provenances étudiées. Equateur (Salinas, T3) a la plus grande hauteur tandis que la plus faible hauteur est détenue par Hyderabad 1 (T8). Les provenances Congo Démocratique (LA23C3V, T1) et Inde (Hyderabad 1, T8) sont différentes du point de vue croissance et développement de la provenance Equateur (Salinas). Quant aux autres provenances, il n'existe pas de différence significative entre elles (Tableau 3).

Diamètre au collet et nombre de ramifications des accessions après un an de culture

L'analyse statistique montre qu'il existe une différence très hautement significative entre les provenances en ce qui concerne le diamètre au collet (Tableau 4). Par rapport au nombre de ramifications, l'analyse statistique montre une différence hautement significative entre provenances.

En comparant les valeurs du diamètre au collet, la provenance Equateur (Salinas, T3) vient en tête suivie des provenances Inde (Hyderabad 2, T7), Sénégal (T6), Cambodge (T4), Kivu (République Démocratique du Congo, T5) et Abomey-Calavi (T9). Les provenances République Démocratique du Congo (LA23C3V, T1) et Inde (Hyderabad 1, T8) ont le diamètre au collet le plus faible des accessions étudiées.

Pour la variable nombre de ramifications, c'est la provenance Inde (Hyderabad 1, T8) qui a enregistré la valeur la plus faible par rapport aux provenances Kivu (République Démocratique du Congo, T5) et Sénégal (T6). Par rapport aux autres provenances, la différence n'est pas significative.

\section{Largeur et longueur des feuilles des accessions}

L'analyse du Tableau 5 montre qu'il existe une différence très hautement significative (au seuil de $0,1 \%$ ) entre les 9 provenances pour les variables largeur et longueur des feuilles. La provenance Equateur (Salinas) a la plus large et la plus longue feuille. Quant aux provenances Sénégal et Abomey-Calavi, elles ont les feuilles les plus petites. En ce qui concerne les autres provenances, les feuilles ont pratiquement les mêmes dimensions.

\section{Poids moyen des graines par provenance}

Le Tableau 6 montre que le poids moyen des graines varie d'une provenance à une autre. Les plants provenant de l'équateur portent les graines les plus lourdes en moyenne. Mais ces plants sont également les plus tardifs avec une faible production.

Par rapport au nombre de lobes, il est invariable d'une accession à une autre et est égal au nombre de cinq. Les lobes sont aussi peu profonds. En ce qui concerne les caractères qualitatifs étudiés, toutes les accessions ont des feuilles alternes, glabres, pentagonales et de couleur verte comme les pétioles. Par contre, les graines sont de couleur noire. 
Tableau 1 : Provenances des accessions et années de récolte.

\begin{tabular}{llc}
\hline $\mathbf{N}^{\mathbf{0}}$ d'ordre & Provenances & Années de récolte \\
\hline 1 & & 2008 \\
2 & Abomey-Calavi (Bénin) & 2006 \\
3 & Cambodge & 2008 \\
4 & Salinas (Equateur) & 2007 \\
5 & Hyderabad 1(Inde) & 2007 \\
6 & Hyderabad 2 (Inde) & 2006 \\
7 & LA22C3IV Kivu (République Démocratique du Congo) & 2008 \\
8 & Madagascar & 2008 \\
9 & LA23C3V (République Démocratique du Congo) & 2006 \\
\hline
\end{tabular}

Tableau 2 : Taux de germination en fonction des accessions.

\begin{tabular}{llcccc}
\hline $\begin{array}{l}\mathbf{N}^{\mathbf{0}} \\
\text { d'ordre }\end{array}$ & Accessions & $\begin{array}{c}\text { Années } \\
\text { de récolte }\end{array}$ & $\begin{array}{c}\text { Nombre de } \\
\text { semences } \\
\text { semées }\end{array}$ & $\begin{array}{c}\text { Nombre de } \\
\text { graines } \\
\text { germées }\end{array}$ & $\begin{array}{c}\text { Taux de } \\
\text { germination } \\
(\%)\end{array}$ \\
\hline 1 & Abomey-Calavi (Bénin) & 2008 & 15 & 14 & 93 \\
2 & Cambodge & 2006 & 15 & 13 & 87 \\
3 & Salinas (Equateur) & 2008 & 14 & 13 & 87 \\
4 & Hyderabad 1(Inde) & 2007 & 15 & 5 & 33 \\
5 & Hyderabad 2 (Inde) & 2007 & 14 & 5 & 33 \\
6 & LA22C3IV (Kivu) & 2006 & 13 & 10 & 67 \\
7 & Madagascar & 2008 & 15 & 13 & 87 \\
8 & LA23C3V (Congo) & 2008 & 15 & 13 & 87 \\
9 & Sénégal & 2006 & 15 & 5 & 33 \\
\hline
\end{tabular}

Tableau 3 : Hauteur moyenne des accessions après un an de culture.

\begin{tabular}{lc}
\hline Traitements (provenances) & Hauteur moyenne des plants (cm) \\
\hline T1 (LA23C3V (Congo Démocratique) & $156,40 \pm 7,7 \mathrm{~b}$ \\
T2 Madagascar & $178,20 \pm 7,8 \mathrm{ab}$ \\
T3 Salinas (Equateur) & $202,40 \pm 14,7 \mathrm{a}$ \\
T4 Cambodge & $174,40 \pm 5,1 \mathrm{ab}$ \\
T5 LA22C3IV (Kivu ) & $182,40 \pm 6,7 \mathrm{ab}$ \\
T6 Sénégal & $174,40 \pm 6,9 \mathrm{ab}$ \\
T7 Hyderabad2 (Inde) & $186,20 \pm 11 \mathrm{ab}$ \\
T8 Hyderabad 1 (Inde) & $153,80 \pm 6,6 \mathrm{~b}$ \\
T9 Abomey-Calavi & $177,40 \pm 8,9 \mathrm{ab}$ \\
Probabilité & $0,02 *$ \\
cv\% & 11,2 \\
\hline Les moyennes suivies de la même lettre ne sont pas significativement différentes au seuil de 5\% $; *=$ différence significative \\
au seuil de 5\% cv = coefficient de variation. T1 = provenance République Démocratique du Congo ; T2 = Madagascar, T3 \\
$=$ Equateur ; T4 = Cambodge ; T5 = Kivu (République Démocratique du Congo) ; T6 = Sénégal ; T7 = Inde (Hyderabad 2); \\
T8 = Inde (Hyderabad 1) et T9 = Abomey-Calavi.
\end{tabular}


Tableau 4 : Diamètre au collet et nombre de ramifications après un an de culture.

\begin{tabular}{lcc}
\hline Traitements (provenances) & $\begin{array}{c}\text { Diamètre moyen au } \\
\text { collet }(\mathbf{m m})\end{array}$ & $\begin{array}{c}\text { Nombre moyen de } \\
\text { ramifications }\end{array}$ \\
\hline T1 (LA23C3V (Congo Démocratique) & $50,40 \pm 1,2 \mathrm{c}$ & $7,0 \pm 1,2 \mathrm{ab}$ \\
T2 Madagascar & $57,80 \pm 1,5 \mathrm{~b}$ & $6,60 \pm 1,5 \mathrm{ab}$ \\
T3 Salinas (Equateur) & $67,20 \pm 1,7 \mathrm{a}$ & $6,6 \pm 0,9 \mathrm{ab}$ \\
T4 Cambodge & $60,0 \pm 1,2 \mathrm{~b}$ & $6,80 \pm 0,5 \mathrm{ab}$ \\
T5 LA22C3IV (Kivu ) & $60,0 \pm 1,7 \mathrm{~b}$ & $10,8 \pm 1,8 \mathrm{a}$ \\
T6 Sénégal & $60,2 \pm 0,86 \mathrm{~b}$ & $10,8 \pm 1,8 \mathrm{a}$ \\
T7 Hyderabad2 (Inde) & $61,0 \pm 3,03 \mathrm{~b}$ & $7,20 \pm 1,5 \mathrm{ab}$ \\
T8 Hyderabad 1 (Inde) & $51,0 \pm 2,5 \mathrm{c}$ & $5,20 \pm 0,7 \mathrm{~b}$ \\
T9 Abomey-Calavi & $58,0 \pm 0,9 \mathrm{~b}$ & $10,0 \pm 1,05 \mathrm{ab}$ \\
Probabilité & $<0,0001 * * *$ & $0,009 * *$ \\
cv\% & 6,7 & 3,4 \\
\hline Les moyennes suivies de la même lettre ne sont pas significativement différentes au seuil de $5 \% ; * *=$ différence hautement \\
significative au seuil de 1\%; *** = différence très hautement significative au seuil de $0,1 \% ; \mathrm{cv}=$ coefficient de variation.
\end{tabular}

Tableau 5 : Largeur et longueur des feuilles des accessions.

\begin{tabular}{lcc}
\hline Traitements (provenances) & $\begin{array}{c}\text { largeur moyenne } \\
\text { des feuilles }(\mathbf{c m})\end{array}$ & $\begin{array}{c}\text { longueur moyenne des } \\
\text { feuilles }(\mathbf{c m})\end{array}$ \\
\hline T1 (LA23C3V (Congo Démocratique) & $14,53 \pm 0,3 \mathrm{~b}$ & $13,22 \pm 0,5 \mathrm{~b}$ \\
T2 Madagascar & $14,49 \pm 0,2 \mathrm{~b}$ & $13,10 \pm 0,14 \mathrm{~b}$ \\
T3 Salinas (Equateur) & $21,27 \pm 0,8 \mathrm{a}$ & $17,38 \pm 0,6 \mathrm{a}$ \\
T4 Cambodge & $13,16 \pm 0,5 \mathrm{bc}$ & $11,90 \pm 0,5 \mathrm{bc}$ \\
T5 LA22C3IV (Kivu ) & $13,15 \pm 0,3 \mathrm{bc}$ & $12,08 \pm 0,3 \mathrm{bc}$ \\
T6 Sénégal & $12,39 \pm 0,4 \mathrm{c}$ & $11,21 \pm 0,4 \mathrm{c}$ \\
T7 Hyderabad2 (Inde) & $13,45 \pm 0,3 \mathrm{bc}$ & $12,42 \pm 0,2 \mathrm{bc}$ \\
T8 Hyderabad 1 (Inde) & $13,85 \pm 0,3 \mathrm{bc}$ & $12,0 \pm 0,4 \mathrm{bc}$ \\
T9 Abomey-Calavi & $12,59 \pm 0,5 \mathrm{c}$ & $11,29 \pm 0,4 \mathrm{c}$ \\
Probabilité & $<0,0001 * * *$ & $<0,0001 * * *$ \\
cr\% & 9,5 & 10,2 \\
\hline Les moyennes suivies de la même lettre ne sont pas significativement différentes au seuil de $5 \% ; * * *$ différence très \\
hautement significative au seuil de $0,1 \%$; cv = coefficient de variation.
\end{tabular}


Tableau 6 : Poids moyen des graines par provenance.

\begin{tabular}{lccc}
\hline Provenances & $\begin{array}{c}\text { Poids moyen } \\
(\mathbf{g})\end{array}$ & (Minimum & Maximum) \\
\hline T1 (LA23C3V (Congo Démocratique) & 0,649 & 0,519 & 0,727 \\
T2 Madagascar & 0,647 & 0,454 & 0,740 \\
T3 Salinas (Equateur) & 0,749 & 0,703 & 0,783 \\
T4 Cambodge & 0,697 & 0,577 & 0,791 \\
T5 LA22C3IV (Kivu ) & 0,578 & 0,424 & 0,722 \\
T6 Sénégal & 0,512 & 0,340 & 0,681 \\
T7 Hyderabad2 (Inde) & 0,734 & 0,675 & 0,782 \\
T8 Hyderabad 1 (Inde) & 0,577 & 0,462 & 0,759 \\
T9 Abomey-Calavi & 0,579 & 0,411 & 0,708 \\
Cv\% & & 13,3 & \\
\hline cv = coefficient de variation & & &
\end{tabular}

\section{DISCUSSION}

\section{Taux de germination}

La scarification des graines avant leur semis a donné en 7 jours un pourcentage de germination plus élevé que le trempage dans l'eau froide pendant 24 heures préconisé par Gour en 2006. En 7 jours, nous avons obtenu pour les graines de meilleure qualité, un pourcentage moyen de $88 \%$, alors que Gour n'était qu'à $72 \%$ en huit (8) jours. Les résultats obtenus en taux de germination montrent une variation en fonction de la durée et des méthodes de conservation. Lorsque les graines sont nouvellement récoltées, elles ont un pourcentage élevé de germination. $J$. curcas est une plante oléagineuse contenant 43 à 60\% d'huile (Neuwinger, 1994 ; Gandhi et al., 1995 ; Sharma et al.,1997 ; Wink et al., 1997; Gübitz et al., 1999; Beerens, 2007 ; Tewari, 2007). Cette huile végétale dont la composition et les caractéristiques varient en fonction des écotypes est très riche en triglycérides (Makkar et al., 1997 ; Gübitz et al., 1999; Achten et al., 2008). Ces triglycérides peuvent être hydrolysés par les triacylglycérols hydrolases dont les actions sont néfastes pour les graines en stockage car elles sont responsables de leur altération
(Fickers et al., 2008). Cette altération des graines contenant de l'huile par les lipases au cours du stockage est à l'origine des faibles taux de germination enregistrés au niveau de ces dernières lorsqu'elles sont semées après leur déstockage. Les méthodes de conservation sont alors importantes lorsque les graines doivent être conservées pendant une longue durée avant leur mise en germination.

\section{Caractères agromorphologiques}

Les caractères quantitatifs et qualitatifs évalués montrent que les accessions diffèrent d'une provenance à une autre. Toutes les collections cultivées se sont bien comportées du point de vue croissance et développement. Ceci confirme la grande adaptabilité écologique de $J$. curcas rapportée par plusieurs auteurs (Heller, 1996; Foidl et al., 1996 ; Makkar et Becker, 1999 ; Achten et al., 2008). L'apport d'eau aux plants pendant la saison sèche a eu une influence positive sur le développement et la floraison. Cette floraison est restée continuelle confirmant ainsi les travaux de Heller (1996) qui stipulent qu'en régions humides ou sous irrigation, une floraison permanente de $J$. curcas est 
observée toute l'année. En ce qui concerne la hauteur des arbustes, la provenance Equateur (Salinas) a donné la meilleure hauteur, le diamètre au collet le plus grand et la feuille la plus développée (largeur et longueur plus grandes). Il en est de même pour le poids moyen des graines. Mais cette provenance est la moins productive et la plus tardive des accessions (floraison 11 mois après semis).

Le poids moyen enregistré par graine au niveau de toutes les collections varie de 0,512 $\mathrm{g}$ à $0,749 \mathrm{~g}$. Ces résultats sont en accord avec les travaux de Makkar et al. $(1997,1998)$ qui ont trouvé des valeurs variant de 0,490 à $0,860 \mathrm{~g}$ pour une collection d'une vingtaine de provenances. Les travaux de Makkar et collaborateurs ont montré que l'arbuste ayant la graine la plus lourde provient du Nicaragua et celui ayant la graine la plus légère du Cap Vert. Nos travaux et ceux de Makkar et collaborateurs montrent que les écotypes de graines lourdes se trouvent sur le continent Latino-Américain, continent d'origine de $J$. curcas.

Par rapport au nombre de ramifications, les provenances Sénégal et Kivu ont le nombre de ramifications le plus élevé, suivi d'Abomey-Calavi, d'Hydérabad 2 (Inde), du LA23C3V de la République Démocratique du Congo et des autres provenances. Les accessions ayant un nombre de ramifications plus élevé se sont montrées meilleurs producteurs comparés aux autres accessions. C'est le cas de la provenance Sénégal qui non seulement a le nombre de ramifications le plus élevé, mais est la plus précoce (floraison 5 mois après semis). Compte tenu de ces deux caractères, la provenance Sénégal peut être considérée comme un génotype intéressant pouvant être utilisé dans les programmes d'amélioration génétique. Concernant les variables longueur et largeur des feuilles, la provenance Sénégal a également les feuilles aux dimensions les plus réduites avec respectivement $12,39 \mathrm{~cm}$ pour la largeur et $11,21 \mathrm{~cm}$ pour la longueur. Etant donné que cette provenance Sénégal a un feuillage moins dense par rapport aux autres accessions, en culture pure, elle pourrait avoir une densité de plants à l'hectare plus importante que les autres accessions.

Après un an de culture, toutes les provenances ont atteint une hauteur minimale de $150 \mathrm{~cm}$. Une bonne condition de culture pourrait permettre à ces provenances d'atteindre en quelques années la hauteur de 5 à $8 \mathrm{~m}$ évoquée par Aderibigbe et al. (1997) et Achten et al. (2008). Il serait bon pour favoriser les récoltes manuelles et augmenter la production, de procéder à des tailles régulières des arbustes afin d'induire un nombre important de ramifications latérales et autres.

\section{Conclusion}

Ce travail sur la caractérisation agromorphologique des provenances de $J$. curcas cultivées au Bénin a montré une grande variation des caractères étudiés. La provenance Equateur (Salinas) a donné la hauteur la plus élevée, le diamètre au collet le plus grand et la feuille la plus grande. La provenance Sénégal a donné le nombre de ramifications le plus élevé, la feuille ayant les dimensions les plus réduites et une production en fruits plus élevée. Les caractères agromorphologiques évalués ont permis d'identifier parmi les provenances cultivées, les écotypes pouvant rentrer dans un programme d'amélioration et de sélection variétale en vue de mettre à la disposition des producteurs des nouveaux cultivars plus performants et plus précoces. Les provenances susceptibles d'être utilisées sont : Sénégal, Madagascar, Cambodge, Kivu et Hydérabad 2 de l'Inde. Mais il serait bon d'augmenter le nombre des accessions en faisant surtout des prospections dans toute la zone Ouest- Africaine afin d'accroître la collection.

\section{REFERENCES}

Achten WMJ, Verchot L, Franken YJ, Mathijs E, Singh VP, Aerts R, Muys B. 2008. Jatropha bio-diesel production and use. Biomass and Bioenergy, 32(12), 10631084 
Aderibigbe AO, Johson COLE, Makkar HPS, Becker K, Foidl N. 1997. Chemical composition and effect of heat on organic matter and nitrogen-degradability and some antinutritional components of Jatropha meal. A. Fe.ed Sci. Tech., 67: 223-243.

Ajiwé VIE, Okeke CA, Agbo HU, Ogunleye GA, Ekwuozor SC. 1996. Extraction, characterization and industrial uses of velvet-tamarind, physic-nut and nickernutseed oils. Biores.Tech., 57: 297-299.

Arbonnier C. 2002. Arbres, Arbustes et Lianes des Zones Sèches d'Afrique de l'Ouest ( $2^{\text {ème }}$ édition). CIRAD MAM : France.

Beerens P. 2007. Screw-pressing of Jatropha seeds for fuelling purposes in developed countries. MSc dissertation, Eindhoven University of Technology, Eindhoven, p. 63.

de Souza S. 2005. Guide Pratique de Phytothérapie: 100 Plantes Médicinales du Bénin. Cotonou.

Fickers P, Destain J, Thonart P. 2008. Les lipases sont des hydrolases atypiques: principales caractéristiques et applications. Biotechnol. Agron. Soc. Enriron., 12(2): 119-130.

Foidl N, Foidl G, Sanchez M, Mittelbach M, Hackel S. 1996. Jatropha curcas L. as a source for the production of biofuel in Nicaragua. Biores. Tech., 58: 77-82.

Gandhi VM, Cherian KM, Mullky MJ. 1995. Toxicological studies on ratanjyot oil. Food Chem. Toxicol., 33: 39-42.

Gour VK. 2006. Production practices including post-harvest management of Jatropha curcas. In Proceedings of the Biodiesel Conference Toward Energy Independence-focus of Jatropha, Singh B, Swaminathan R, Ponraj V (eds). Hyderabad: New Delhi, Rashtrapati Bhawan; 223-251.

Grim C. 1999. Evaluation of damage to physic nut (Jatropha curcas L.) by true bugs. Ent. Exp. Ap., 92: 127-136.

Gübitz GM, Mittelbach M, Trabi M. 1999. Exploitation of the tropical oil seed plant
Jatropha curcas L. Biores. Tech., 67: 7382.

Heller J. 1996. Physic Nut. Jatropha curcas L. Promoting the conservation and use of underutilized and neglected crops; Institute of Plant Genetics and Crop Plant Research (Gartersleben) and International Plant Genetic Resources Institute: Rome; Vol.1.

Henning RK. 1997. Fuel production improves food production: The Jatropha project in Mali. In Biofuels and Industrial Products from J. curcas. Gübitz GM, Mittelbach M, Trabi M (eds). DBV Graz; 92-97.

Liu SY, Sporer F, Wink M, Jourdane J, Henning R, Li y L, Ruppel A. 1997. Anthraquinones in Rheum palmantum and Rumex dentatus (Polygonanceae), and phorbol esters in Jatropha curcas (Euphorbiaceae) with molluscicidal activity against the schistosome vector snails Oncomelania, Biomphalaria and Bulinus. Trop. Med. Int. Health, 2: 179188.

Makkar HPS, Becker K, Sporer F, Wink M. 1997. Studies on nutritive potential and toxic constituents of different provenances of Jatropha curcas. J. Agric. Food Chem., 45: 3152-3157.

Makkar HPS, Aderibigbe AO, Becker K. 1998. Comparative evaluation of nontoxic and toxic varieties of Jatropha curcas for chemical composition, digestibility, protein degradability and toxic factors. Food Chem., 62: 207-215.

Makkar HPS, Becker K. 1999. Plant toxins and detoxification methods to improve feed quality of tropical seeds Review. Asian-Aus. J. Anim. Sci., 12(3): 467-480.

Neuwinger HD. 1994. Afrikanische Arzneipflanzen und Jagdgifte. WV Gesmh, 450-457.

Nwosu MO, Okafor JI. 1995. Preliminary studies of the antifungal activities of some medicinal plants against Basidiobolus and some other pathogenic fungi. Mycoses, 38: 191-195.

Scherchan DP, Thapa YB, Khadka RJ, Tiwari TP. 1989. Effect of green manure on rice 
production. Pakhribas Agricultural Centre, Occasional paper 2, p.12.

Sharma GD, Gupta SN, Khabiruddin M. 1997. Cultivation of Jatropha curcas as a future source of hydrocarbon and other industrial products. In Biofuels and Industrial Products from Jatropha curcas. Gübitz GM, Mittelbach M, Trabi M (eds). DBV Graz; 19-21.

Solsoloy AD, Solsoloy TS. 1997. Pesticidal efficacy of formulated J. curcas oil on pests of selected field crops. In Biofuels and Industrial Products from Jatropha curcas, Gübitz GM, Mittel-bach M, Trabi M (eds). DBV Graz; 216-226.
Staubmann R, Schubert-Zsilavecz M, Hiermann A, Kartnig T. 1997. The antiinflammatory effect of J. curcas leaves. In Biofuels and Industrial Products from Jatropha curcas. Gübitz GM, Mittelbach M, Trabi M (eds). DBV Graz; 60-64.

Tewari DN. 2007. Jatropha and Biodiesel $\left(1^{\text {st }}\right.$ edition). Ocean books Ltd: New Delhi.

Wink M, Koschmieder C, Sauerwein M, Sporer F. 1997. Phorbol esters of $J$. curcas - biological activities and potential applications. In Biofuels and Industrial Products from Jatropha curcas. Gübitz GM, Mittelbach M, Trabi M (eds). DBV Graz; 160-166. 